\title{
Modeling MOOC Student Behavior With Two-Layer Hidden Markov Models
}

\author{
Chase Geigle $^{1} \quad$ ChengXiang Zhai $^{1}$ \\ ${ }^{1}$ Department of Computer Science \\ University of Illinois at Urbana-Champaign \\ Urbana, Illinois, USA \\ \{geigle1, czhai\}@illinois.edu
}

\begin{abstract}
Massive open online courses (MOOCs) provide educators with an abundance of data describing how students interact with the platform, but this data is highly underutilized today. This is in part due to the lack of sophisticated tools to provide interpretable and actionable summaries of huge amounts of MOOC activity present in log data. In this paper, we propose a method for automatically discovering student behavior patterns by leveraging the click log data that can be obtained from the MOOC platform itself in a completely unsupervised manner.
\end{abstract}

\section{Author Keywords}

MOOC log analysis; student behavior modeling; Markov models; hidden Markov models

\section{CCS CONCEPTS}

-Mathematics of computing $\rightarrow$ Kalman filters and hidden Markov models; •Computing methodologies $\rightarrow$ Mixture modeling;

\section{INTRODUCTION}

The proliferation of massive open online courses (MOOCs) has resulted in a profound impact on education. As more and more learners turn to MOOCs to educate themselves on various topics, more and more behavioral data is being collected as part of the system on which the MOOC is offered. The data present in these logs has the power to aid us in understanding the behavior of students who take our MOOCs, which is mostly undetectable for instructors of these MOOCs today due to its vast scale. As a result, the rich data available through these MOOC logs is highly underutilized today.

What stands in the way? Clearly, intelligent systems to create concise and digestible summaries of the massive amount of interaction data collected are needed in order to empower the instructors of these courses. If we can understand how users are interacting with our MOOCs, we are much more likely to be able to make changes to these courses that positively impact learners. While we can easily observe the changes in behavior of students in real classrooms, MOOCs present

Permission to make digital or hard copies of all or part of this work for personal or classroom use is granted without fee provided that copies are not made or distributed for profit or commercial advantage and that copies bear this notice and the full citation on the first page. Copyrights for components of this work owned by others than the author(s) must be honored. Abstracting with credit is permitted. To copy otherwise, or republish, to post on servers or to redistribute to lists, requires prior specific permission and/or a fee. Request permissions from permissions@acm.org. $L @ S$ 2017, April 20-21, 2017, Cambridge, MA, USA.

Copyright is held by the owner/author(s). Publication rights licensed to ACM. ACM 978-1-4503-4450-0/17/04 . .\$15.00.

DOI: http://dx.doi.org/10.1145/3051457.3053986 a challenge due to their hands-off nature and sometimes irregular schedule due to being a full-time worker. We view this paper as attempting to bridge this gap. Specifically, in this paper we propose unsupervised learning methods for automatically discovering and characterizing student learning behavior patterns or profiles from large collections of click logs associated with MOOCs.

Our work is motivated by the following observations:

1. Student behavior is complicated and cannot necessarily be captured sufficiently by rule-based methods such as those explored by Kizilcec et al. [5] and Davis et al. [1]. We instead propose to treat student behavior patterns as being characterized (represented) via a sequence of latent states. This allows us to automatically capture patterns that we might not have been able to articulate clearly a priori via a series of rules, and also allows us to model the inherent uncertainty in assigning a student's behavior to a particular pattern or group.

2. Student behavior can vary over time. Previous models that treat students as exhibiting only one behavioral pattern over time [2] miss out on the opportunity to understand student behavior dynamics in a course. We propose a latent space model with latent state transitions to flexibly model the dynamics.

3. Analysis of student behavior can and should be performed at varying levels of granularity. This requires us to aggregate data over time with different levels of resolution; existing models tend to come with a particular assumption about the resolution of time they consider $[2,5,8]$. We propose a more flexible model to accommodate different levels of resolution.

Thus, what we propose is a latent variable approach to mining student behavior patterns that is probabilistic for inference and flexible to model state changes over different time resolutions. More specifically, we propose a novel two-layer hidden Markov model (TL-HMM) to discover latent student behavior patterns via unsupervised learning on large collections of student behavior observation sequences. Evaluation results on a MOOC data set on Coursera demonstrate that TL-HMM can effectively discover a variety of interesting interpretable student behavior patterns at different levels of resolution, many of which are beyond what existing approaches can discover. TL-HMM further enables easy use of the discovered patterns for discriminative analysis such as prediction of learning outcome. Since our proposed methods are unsupervised, they can potentially be applied to any MOOC data without 
requiring any manual work to facilitate understanding of student behaviors and their variations, opening up many possibilities for developing intelligent tutoring systems that can adaptive to student behavior.

\section{A TWO-LAYER HMM FOR MOOC LOG ANALYSIS}

Our general idea is to use a probabilistic generative model to model the student activities as recorded in a MOOC log, which means we will assume that all the observed student activities are samples drawn (i.e., "generated") from a parameterized probabilistic model. We can then estimate the parameter values of the probabilistic model by fitting the model to a specific MOOC log data set. The estimated parameter values could then be treated as the latent "knowledge" discovered from the data. Because such a generative model attempts to fit all the data, it enables us to discover interesting patterns that can explain the overall behavior of a student or the common behavior patterns shared by many students.

An HMM is a specific probabilistic generative model with a "built-in" state transition system that would control the data to be generated by the model, thus it is especially suitable for modeling sequence data [7]. At any moment, the HMM would be in one of $k$ states $U=\left\{u_{1}, \ldots, u_{k}\right\}$, and at the next moment, the HMM would move to another state stochastically according to a transition matrix that specifies the probability of going to state $u_{i}$ when the HMM is currently in state $u_{j}$, i.e., $p\left(u_{i} \mid u_{j}\right)$. When the HMM is in state $u$, the HMM can generate an observable data point $x$ according to an output probabilistic model $p(x \mid u)$. Thus if we "run" an HMM for $N$ time points denoted by $t=1, \ldots, N$, the HMM could "generate" a sequence of observations $x_{1} \ldots x_{N}$, where each $x_{i}$ is an output symbol by going through a sequence of hidden states $w_{1} \ldots w_{N}$ where $w_{i} \in U$ is a state. The association of such a latent sequence of state transitions with the observed symbols makes it possible to use HMM to "decode" the latent behavior of students behind the surface behavior we directly observe in the log data, allowing for understanding student behavior more deeply than a model with no latent state variables.

In many ways, the generation process behind an HMM is meant to simulate the actual behavior of a student. We may say that students transition through different "task states" (or "behavior states") in the process of study. One such task state may be to learn about a topic by mostly watching lecture videos, another task state may be to work on quizzes, and yet another may be to participate in forum discussions. While in each of these different states, the student would tend to exhibit different surface "micro" behaviors. For example, in the lecture study state, the student would tend to have many videowatching related behaviors and occasionally forum activities, while in the quiz-taking state (in order to pass each module), the student would tend to show many quiz-related "micro" activities as well as asking questions or checking discussions on the forum. Note that due to the complexity of the student behavior, it is very difficult to accurately prescribe the specific surface "micro" behavior patterns for each state in advance, especially without prior knowledge about the students. For example, forum activities are likely interleaved with other activities in every task state and the interleaving pattern can be somewhat irregular with potentially many variations. The major motivations for using an HMM are that (1) it uses a probabilistic model (i.e., the output probability distribution $p(x \mid u)$ conditioned on each state) to directly capture the inevitable uncertainty in the association of surface "micro" activities with their corresponding latent task/behavior state, which is often our main target to discover and characterize, and (2) it does not make any assumption about which latent task/behavior state must be associated with which observed activities or how a student would move from one state to another, but instead allows our data to "tell" us what kind of associations are most likely, what kind of transitions are most probable, and which states tend to be more long-lasting for any particular set of students.

However, if we use an ordinary HMM to analyze our data, we would treat each observed "micro" activity (e.g., video watching, or forum post reading) as an output symbol, and thus the output distribution $p(x \mid u)$ for each discovered latent state would be a simple distribution over all kinds of observable micro activities recorded in our log data (e.g., $50 \%$ lecture watching, $8 \%$ quiz taking, $7 \%$ quiz submission, $2 \%$ course wiki reading, ...). While such a distribution is meaningful and can already help us interpret the corresponding latent state, it only gives us a rather superficial characterization of student behavior.

Ideally, we want $p(x \mid u)$ to characterize the directly observable "micro" behavior in more detail to further capture the relations and dependencies of these micro activities. To this end, we would treat an entire sequence of micro activities (e.g., one session of activities) as an observed "symbol" from a latent state, and further model the generation of such a sequence with another Markov model where we treat each micro activity as an observable state, and model the transitions between these activity states in very much the same way as the state transitions in HMM.

Adding this second layer would allow us to characterize a latent task state in much more detail, as it would reveal not only what activities are most common to a task state, but also the transition patterns between these "micro" activities (e.g., it can reveal frequent back-and-forth transitions between quiz-taking and quiz-submission, which would suggest a concentrated period of taking quizzes). Combining this "surface" Markov model over the "micro" actions with the "deep" hidden Markov model over the latent task states gives us a fairly general and powerful two-layer HMM (TL-HMM) that can simultaneously learn "deeply" the latent task/behavior states and their transitions as well as the corresponding "micro" activity transition patterns associated with each latent state to facilitate interpretation and analysis of the discovered latent state patterns. Our implementation of the learning algorithm for TL-HMMs is included as part of the META toolkit [6].

\section{EXPERIMENT RESULTS}

As an analysis tool, the TL-HMM model provides with us the following two patterns to characterize student behavior: (1) the latent state representations, and (2) the latent state transitions. Thus to evaluate the proposed model, we conduct 
experiments to qualitatively analyze both types of patterns discovered from empirical MOOC log data.

Specifically, we looked at the MOOC logs associated with the textretrieval-001 Coursera MOOC offered by UIUC and extracted a dataset consisting of 18,941 students who produced 85,240 sequences with an average length of 7.31. We used the following ten actions as our action set A: (1) quiz start, (2) quiz submit, (3) wiki (course material), (4) forum list (view the list of all forums), (5) forum thread list (view the list of all threads in a specific forum), (6) forum thread view (view the list of posts within a specific thread), (7) forum search (a search query issued against the forum), (8) forum post thread (a new thread was posted), (9) forum post reply (a new post was created within an existing thread), and (10) view lecture (defined as either streaming or downloading a lecture video).

\section{Latent State Representations}

To visualize these Markov models that represent our latent states, we plot them as a directed graph where we set the size of a node to be proportional to its probability of being visited during a random walk. We let the thickness of a directed edge $(u, v)$ reflect the probability of taking that edge given that a random walk is currently at note $u$ (as indicated by the transition matrix) ${ }^{1}$.

Figure 1 shows the latent state representations learned by fitting a 4-state TL-HMM to the textretrieval-001 sequence dataset. Our interpretations of the states is as follows: (a) state 0 likely captures all sequences where a student logged in to the platform and did nothing else (likely just checking for updates); (b) state 1 seems to capture a more engaged browsing session, where there is non-negligible probability associated with different activities such as quiz taking and forum browsing and, importantly, these activities have high probability symmetric edges (so students are taking quizzes one after the other, or viewing forum threads in succession); (c) state 2 captures a "forum browsing" state, with most weight being placed on consecutive thread views; and (d) state 3 seems to capture a more passive student, with negligible probability mass associated with forum activity (with low symmetry in the edges). The link between "quiz submit" and "quiz start" (indicating quiz repetition) is also significantly lower than state 1 .

\section{Transitions Between Latent States}

A unique property of our model is its ability to capture transitions between the behavior patterns themselves that are captured by the latent states. In Figure $2 \mathrm{a}$ we show the latent state transition diagram for a 4-state TL-HMM fit on textretrieval-001. We can immediately observe two things: (1) each latent state has a very high "staying" probability, and (2) the prevalence of each latent state matches our intuition. In particular, we can see that the forum browsing state (state 2) has relatively lower probability than the other states as we might expect. It also makes sense that state 0 (low activity) has rather high probability. If we look at state 1 and state 3 ,

\footnotetext{
${ }^{1}$ We do not plot the transition probabilities directly within the figure to ease readability; we instead will mention relevant transition probabilities in the text as we discuss the plots. The plots were created using python-igraph: http://igraph.org/python/.
}

their relative probabilities match our intuition as well: there should be more students exhibiting more passive behaviors (state 3 ) than very active behaviors (state 1).

Thus, we might expect to see students that perform well in the course preferring states 1 and 2 over states 0 and 3 . To verify this, we took the model we learned on the full training data and retrofit it to training data consisting only of sequences produced by students in textretrieval-001 that had perfect marks. To prevent the latent state meanings from drifting, we forced the model parameters associated with their Markov model representations to be fixed, in effect only learning initial and transition probabilities for the top layer of our TL-HMM. We show the updated latent state transition diagram in Figure 2b. We can clearly see that the probability of state 2 has increased dramatically, consistent with previous observations of the positive correlation between forum activity and grades [3], while the probability of states 0 and 3 has decreased. State 1 had its probability increase very slightly.

In Figure $2 \mathrm{c}$ we plot the latent state transition diagram for a second group of "low" students. These students were selected so that they attempted all required quizzes in the course, but such that their average quiz score was $\leq 70 \%$. Here, we see that state 1 has a large increase in size, where we might have expected state 3 to grow instead. However, there is an alternative explanation for this phenomenon. Since state 1 seems to indicate a highly engaged student, it is a perfectly reasonable explanation for the "low" student group as they are going to be working hard to try to fill in the gaps in their knowledge. By contrast, the "perfect" student group likely has many members who can take the quiz more passively and get perfect marks, perhaps because they already know much of the material being presented, or are just naturally strong and do not require much background review to perform well. This also explains why state 1 did not increase in size for the "perfect" group like we were anticipating. Kizilcec et al. [4] observe similar phenomena with the courses they studied where they find that certificate earning is negatively correlated with help seeking behavior. Our model enables data-driven discovery of potentially counter-intuitive insights like this.

\section{CONCLUSION}

We proposed a two-layer hidden Markov model for MOOC student behavior modeling in an unsupervised learning framework on large collections of actions extracted from MOOC $\log$ data. This model is different from existing methods in that it treats behavior patterns as a sequence of latent states, rather than assigning these states in a rule-based manner. It captures the variable behaviors of students over time, and allows analysis at different levels of granularity.

We showed that such a model does in fact capture meaningful behavior patterns and produces descriptions of these behavior patterns that are easy to interpret. We argued that it is important to capture student behavior patterns with more sophisticated models than simple discrete distributions over actions in order to capture information present in bigrams of actions (or above). Finally, we investigated whether we can detect differences in student behavior patterns as they correlate with course performance. Specifically, we demonstrated 


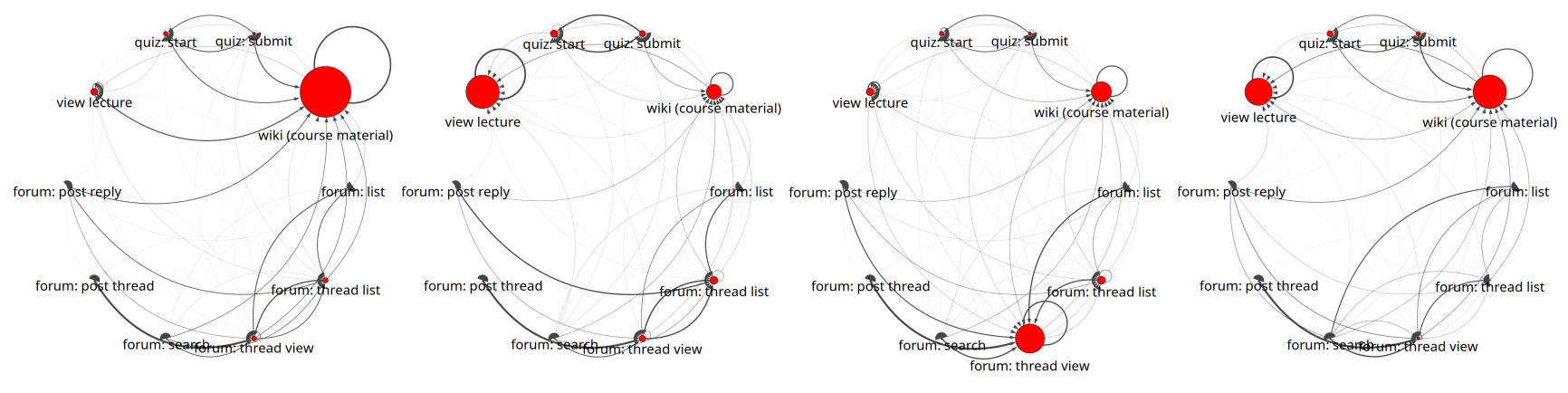

(a) State 0

(b) State 1

(c) State 2

(d) State 3

Figure 1. Example states learned by a 4-state TL-HMM.

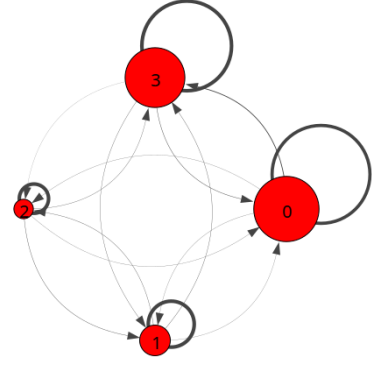

(a)

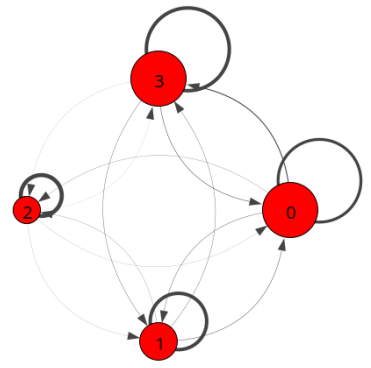

(b)

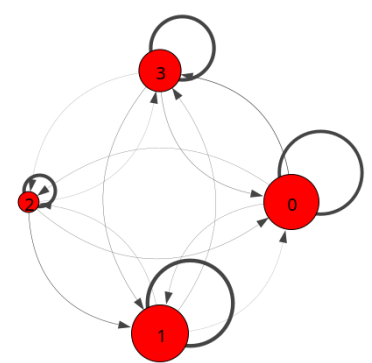

(c)

Figure 2. The latent state transition diagrams for a 4-state TL-HMM fit to textretrieval-001 for all students (a) compared to only "perfect" students (b) and only "low" students (c).

that high-performing students produce substantially different HMM transition diagrams that tend to show longer concentration span in quiz-taking and more active forum participation as compared with the average students.

\section{Future Work}

Although we only experimented with our model on one MOOC, the model is completely general and can be easily applied to analyze the log of any other course to enable deep understanding of student behaviors as well as the coorrelations of such behaviors as other variables such as grades. We plan to develop a MOOC log analysis system based on the proposed model to both facilitate education research and help instructors improve course design.

Specifically, our model can be used to produce an ordered list of latent states for each student over time. Given these labeled sequences, we could correlate course-wide drifts in these latent states with events in a course. For example, we might be able to automatically discover difficult or confusing parts of a course by noticing spikes in the distribution of students over latent states over time.

\section{ACKNOWLEDGMENTS}

This material is based upon work supported by the National Science Foundation under Grant Number DGE-1144245 (GRFP) and Grant Number 1629161.

\section{REFERENCES}

1. Dan Davis, Guanliang Chen, Claudia Hauff, and GeertJan Houben. 2016. Gauging MOOC Learners' Adherence to the Designed Learning Path. In Proc. EDM. 54-61.

2. Louis Faucon, Lukasz Kidzinski, and Pierre Dillenbourg. 2016. Semi-Markov model for simulating MOOC students. In Proc. EDM. 358-363.

3. Jonathan Huang, Anirban Dasgupta, Arpita Ghosh, Jane Manning, and Marc Sanders. 2014. Superposter Behavior in MOOC Forums. In Proc. L@S. 117-126.

4. René F. Kizilcec, Mar Pérez-Sanagustín, and Jorge J. Maldonado. 2017. Self-regulated learning strategies predict learner behavior and goal attainment in Massive Open Online Courses. Computers \& Education 104 (2017), 18 - 33.

5. René F. Kizilcec, Chris Piech, and Emily Schneider. 2013. Deconstructing Disengagement: Analyzing Learner Subpopulations in Massive Open Online Courses. In Proc. LAK. 170-179.

6. Sean Massung, Chase Geigle, and ChengXiang Zhai. 2016. MeTA: A Unified Toolkit for Text Retrieval and Analysis. In Proc. ACL Sys. Demo. 91-96.

7. Lawrence R. Rabiner. 1990. Readings in Speech Recognition. Chapter A Tutorial on Hidden Markov Models and Selected Applications in Speech Recognition, 267-296.

8. Benjamin Shih, Kenneth R Koedinger, and Richard Scheines. 2010. Unsupervied Discovery of Student Strategies. In Proc. EDM. 201-210. 